\title{
Radiologic Staging of Lung Carcinoma: A Report Generator and Database System
}

\author{
Morgan G. Dunne and Sean B. Dunne
}

\begin{abstract}
A checklist used by the radiologist interpreting images on lung carcinoma patients for tumor staging is described. The checklist data is subsequently entered into a user friendly microcomputer program which radiologically stages lung carcinoma according to the updated TNM system, maintains an upgradable relational database and generates printed reports for tumor board meetings.

- 1989 by W.B. Saunders Company
\end{abstract}

KEY WORDS: Lung, carcinoma, cancer staging, computers, software, radiology, databases.

$\mathbf{I}$ $\mathrm{N}$ AN effort to improve, systematize, and standardize the reporting of the radiologic evaluation of lung carcinoma, a simple checklist was developed for use by the interpreting radiologist in conjunction with a custom software system written for microcomputers in dBASE III Plus TM (Ashton Tate, Torrance, CA). The checklist (Fig 1) allows the radiologist to rapidly and systematically record the cogent radiologic findings applicable to tumor staging according to the recently updated TNM system. ${ }^{1-3}$ The radiologic data from the checklist (Fig 1) are easily entered into a user-friendly menu-driven relational database using a series of three digit codes, each of which indicates a specific radiologic finding. The system allows the automatic generation of printed reports for tumor board meetings and maintains an upgradable relational database on all entered lung carcinoma patients.

The checklist (Fig 1) is completed by the radiologist performing a review of available imaging studies (including nuclear medicine scans) pertinent to a new lung carcinoma patient. Further examinations on that patient can be reviewed and additional checklist(s) completed as the pre-treatment workup progresses. Data from all checklists are input into the relational database. As shown in Fig 1, the checklist is

From the Department of Radiology, Scott \& White, Temple, $T X$.

Address reprint requests to Morgan G. Dunne, $M A, M B$, BCh, BAO, Department of Radiology, Scott \& White, 2401 South 31st St, Temple, TX 76508.

(1) 1989 by W.B. Saunders Company

0897-1889/89/0201-0011\$03.00/0 divided into six sections, the first of which records patients data such as hospital identification number (ID\#), name, age, sex, etc.. The next section contains a series of possible lung carcinoma primary locations and the appropriate box or boxes are checked by the radiologist. The three digit codes adjacent to the boxes will subsequently be entered into the microcomputer program for database storage and report generation. The next section enables the radiologist to rapidly record the primary tumor findings that determine the $\mathrm{T}$ classification under the new international TNM staging system. ${ }^{1-3}$ The single digit numbers in braces indicate the $T$ classification related to that specific finding.

The next section deals with radiologic assessment of the lymph node groups germane to determination of the $\mathbf{N}$ classification under the TNM staging system. A number of papers describe computed tomography (CT) evaluation of specific nodal groups in the detection of nodal spread of lung carcinoma. ${ }^{4-9}$ The principal criterion used to imply nodal spread of tumor is nodal enlargement. Various authors have suggested threshold measurements on CT for mediastinal nodes; above which nodal involvement with tumor is suggested. ${ }^{4-9}$ The nodal group mapping system developed by the American Thoracic Society (ATS) and subsequently modified by others was used in the checklist illustrated in Fig $1 .^{2,10}$ The modified ATS system is listed in Tables 1 and 2. A three digit computer code number appears before the ATS descriptor of each nodal group on the checklist (Fig 1). The measurement (in $\mathrm{mm}$ ) after each ATS descriptor indicates the threshold value for the short axis diameter of nodes in that group (Fig 1). ${ }^{4}$ The numbers in braces indicate the $\mathrm{N}$ classification under the TNM system $(2 / 3$ means the $N$ classification is 2 if the nodal group in question is ipsilateral and 3 if contralateral to the primary tumor respectively) (Fig 1). The radiologist using the checklist indicates which nodal groups exceed the short axis diameter threshold by checking the appropriate boxes. Mediastinal nodes of normal size may harbor metastatic deposits and nodes may be enlarged without such deposits. Thus, the 


\begin{tabular}{|c|c|c|}
\hline \multicolumn{3}{|c|}{ Imaging Study Reviev Date: } \\
\hline \multicolumn{3}{|l|}{ PAIIENT DATA: } \\
\hline \multicolumn{2}{|l|}{ LKane: } & \\
\hline \multicolumn{3}{|l|}{ TUMOR LOCATION: } \\
\hline 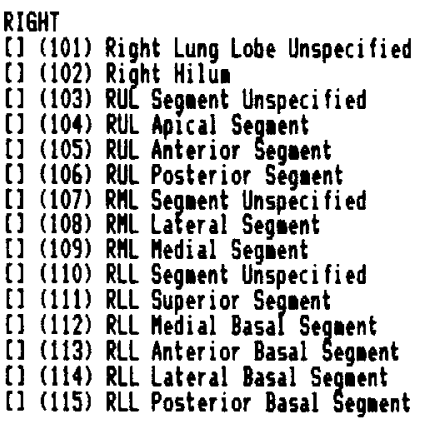 & $\begin{array}{l}\text { LEFT } \\
\text { [] (116) Left Lung Lobe Unspecified } \\
\text { [] (117) Left Hilü } \\
\text { [] (118) LUL Segnent Unspecified } \\
\text { [] (119) LUL Apical-Posterior Segnent } \\
\text { [] (120) LUL Anterior Segnent } \\
\text { [1 (121) LUL Superior Lingular Seguent } \\
\text { [] (122) LUL Inferior Lingular Segnent } \\
\text { [] (123) LLL Segnent Unspecified } \\
\text { [1 (124) LLL Superior Segnent } \\
\text { [] (125) LLL Anteronedial Segnent } \\
\text { [1 (126) LLL Lateral Basal Seguent } \\
\text { [] (127) LLL Posterior Basal Seguent }\end{array}$ & 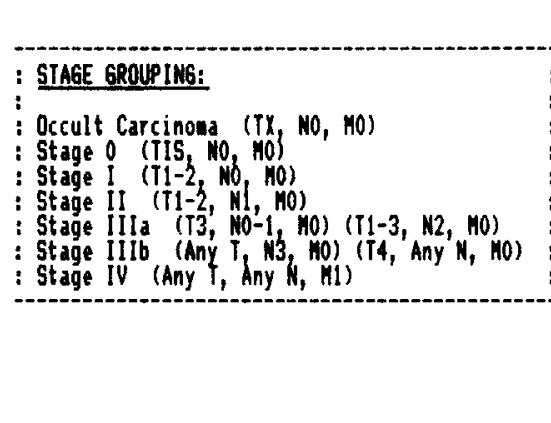 \\
\hline
\end{tabular}

\section{PRIMARY TUMOR ASSESSMEHT (T):}

[] (201) Mediastinal Invasion (4)

[1 (202) Cardiac Invasion \{4\}

[] (203) Great Vessel Invasion (4)

[i (204) Iracheal Invasion (4)

[] (205) Esophageal Invasion (4)

[1 (206) Vertebral Body Invasion \{4\}

[] (207) Carinal Invasion \{4\}

[j (208) Right Pleural Effusion (4)

[] (209) Left Pleural Effusion \{4\}

1] (210) Direct Chest Wall Invasion \{3\}

[1 (211) Direct Diaphragnatic Invasion [3\}
[] (212) Mediastinal Pleural Invasion (3)

[] (213) Tunor <2co Distal to Carina \{3\}

[1] (214) Atelectasis or Obstructive Pneunonitis of Entire Lung (3)

[] (215) Parietal Pericardial Invasion (3)

[1 (216) Visceral Pleural Invasion \{2\}

[] (217) Tunor > $3 \mathrm{c}$ in Dianeter \{2\}

[] (218) Atelectasis or Obstructive Pneunonitis Extending to Hilua but (Entire Lung (2)

[1 (219) Tunor $\langle=3 \mathrm{Cn}$ in Dianeter $\{1\}$

[1 (220) No Evidence of Prialary Tumor $\{0\}$

[1] (221) History of Carcinosa In Situ (IS

[1 (222) History of Bronchopulnonary Secretions Known Positive for haligant Cells $\{x\}$

LYMPH NODE ASSESSMENT (CT Short Axis Dianeter Threshold) [N\}:

RIGHT

[] (301) IR Supraclavicular (10m) \{3\}

[] (311) $2 R$ Upper Paratrach. (7no) $\{2 / 3\}$

[] (312) 4R Lover Paratrach. (10mu) $\{2 / 3\}$

[1] (313) $6 R$ Anterior Mediast. (Ban) $(2 / 3)$

[] (314) $8 R$ Paraesoph. (10ai) $\{2 / 3\}$

[1] (317) LOR Tracheooronchial (10ma) (1/3\}

[1 (318) IIR Intrapul monary (10m) (1/3)

[] (315) 14R Diaphraguatic (10na) $\{2 / 3\}$
LEFT

[] (302) IL Supraclavicular (10a) \{3\}

[] (304) $2 \mathrm{~L}$ Upper Paratrach. (70) \{2/3\}

[] (305) 4L Lover Paratrach. (10ma) \{2/3\}

[i] (306) Et Anterior Mediast. (Ban) $\{2 / 3\}$

[] (307) \&L Paraesoph. (7un) \{2/3\}

[] (308) 10L Peribronchial (7no) $\{2 / 3\}$

[] (309) 11L Intrapulmonary (10mo) $\{1 / 3\}$

[] $(310)$ 14L Diaphragnatic (100n) $\{2 / 3\}$
[] (303) 5 Aortopul wonary (9ma) $\{2 / 3\}$

[1] (316) 7 Subcarinal (11no) $\{2$

[] (319) No Adenopathy $\{0\}$

[] (320) Nodes not Assessed $(x)$

\section{METASTATIC DEPOSIT ASSESSMENT \{M\}:}

\begin{tabular}{|c|c|c|c|}
\hline $\begin{array}{l}\text { [1 (401) Axial Skeleton }\{1\} \\
\text { [f (402) Liver }\{1\} \\
\text { [] (403) Adrenal }\{1\} \\
\text { [] (404) Appendicul ar Skeleton (1\} } \\
\text { [] (405) Brain }\{1\}\end{array}$ & $\begin{array}{l}\text { [1 (406) Spinal Epidural Space }\{1\} \\
\text { [] (407) Spinal Cord }(1) \\
{[](409) \text { Spleen }\{1\}} \\
{[](409) \text { Retroperitoneut }\{1\}} \\
{[](410) \text { Peritoneul }\{1\}}\end{array}$ & $\begin{array}{ll}{[1} & (411) \text { Kidney }\{1\} \\
{[]} & (412) \text { Pancreas }\{1\} \\
{[1} & (413) \text { Ovary (1\} } \\
{[]} & (414) \text { Thyroid }\{1\} \\
{[1} & (415) \text { GI Tract }(1\}\end{array}$ & $\begin{array}{l}\text { [] (416) Lung to Lung (1) } \\
\text { [] (417) No Metastases \{0\} } \\
\text { [] (418) Mets. not Assessed \{w\} }\end{array}$ \\
\hline
\end{tabular}

[] (405) Brain (1)

[] (410) Peritoneus [1]

(415)

[] (418) Hets. not Assessed \{x\}

IMAGING STUDIES REVIEHED:

PLAIN FILH

[1 (501) Chest

[] (502) Abdonen

[i] (503) Pelvis

[] (504) Skull

[1 (505) Spine

[] (506) Upper Linb

[j (507) Lover Liab

CONTRAST

[] (536) Esophagran

[] (537) U6I

[1] (538) Suall Boyel

[] (539) Bariun Enema

[] $(540)$ IVP

[] (541) Sinogran

\section{TOMOGRAPHY}

[] (508) Chest

[i] (509) Abdowen

[] (510) Pelvis

[] (5i1) Skuli

[] (512) Spine

[i (5i3) Upper Linb

[] (514) Lover Linb

ARTERIOGRAPHY

[] (542) Aorta

[] (543) Bronchial

[] (544) Pulconar

[] (545) Head/Neck

[1 (546) Gl/Visceral

[] (547) Extrenity
CT

[1 (515) Chest

[] (5t6) Liver

[] (517) Abdowen

[1 (518) Adrenal

[] (519) Cranial

[1 (520) Pelvis

[] (521) Neck

VENOGRAPHY

[1 (548) Sup. Vena Cava

[] (549) Inf. Vena Cava

[1 (550) Azygogr aphy

[] (551) Head/Neck

[] (552) 6I/Visceral

[] (553) Extrenity
US

[1 (522) Abdowen

[] (523) Liver

[] (524) Kidney

ij (525) Spleen

[1] (526) Pelvis

[] (527) Chest

[i (528) Neck

SPECIALS

[] (554) C-Myelogran

[] (555) T-Myelogran

(1) (556) L-Hyelogy an

[] (557) Chol angiogr as

[] (558) Pyelograt

[] (559) Cystogras
NUC MED

[] (529) Bone

[1 (530) Liver

[] (531) Galliue

[1] (532) $\mathrm{Te}-\mathrm{HIDA}$

[] (533) Thyroid

[] (534) Renograt

[] (535) MUGA Thalliu

HRI

C] (560) Cranial

[] (561) Spinal

[] (562) Chest

[] (563) Abdowen

[] (564) Pelvis

[j (565) Extrenity

Fig 1. Lung carcinoma checklist. 
Table 1. Lymph Node Regions to Right of Midline and Subcarinal Area *

1R: Right supraclavicular or scalene nodes (N3).

2R: Right upper paratracheal nodes to the right of the midline of the trachea between the intersection of the caudal margin of the innominate artery with the right side of the trachea, and the apex of the right lung (N2 if ipsilateral, or N3 if contralateral to primary tumor).

4R: Right lower paratracheal nodes to the right of the midline of the trachea between the cephalic border of the azygos vein and the intersection of the caudal margin of the innominate artery with the right side of the trachea (N2 if ipsilateral, or N3 if contralateral to primary tumor).

6R: Right anterior mediastinal nodes to the right of the midline and anterior to the ascending aorta and innominate artery (N2 if ipsilateral, or N3 if contralateral to primary tumor).

7: Subcarinal nodes caudal to the tracheal carina but within $2 \mathrm{~cm}$ of the carina (N2).

8R: Right paraesophageal nodes to the right of the midline adjacent to the esophagus below region 7 (at least $3 \mathrm{~cm}$ below the carina), in the right pulmonary ligament and posterior diaphragmatic area (N2 if ipsilateral, or N3 if contralateral to primary tumor).

10R: Trachiobronchial nodes to the right of the midline of the trachea from the level of the cephalic surface of the azygos vein and along the right main bronchus to the origin of the right upper lobe bronchus (N1 if ipsilateral, or N3 if contralateral to the primary tumor).

11R: Intrapulmonary nodes along the right lobar or segmental bronchi (distal to regions 7 and 1OR) which are removed with the right lung specimen (N1 if ipsilateral, or N3 if contralatera to the primary tumor)

14R: right anterior diaphragmatic nodes adjacent to the diaphragm at the right cardiophrenic angle and middle diaphragmatic area near the phrenic nerve (N2 if ipsilateral, or N3 if contralateral to the primary tumor).

"Modified American Thoracic Society Classification 2,10

radiologic $\mathbf{N}$ classification will, at times, be false positive or false negative. It is hoped that through the application of this systematic method of nodal evaluation, the true value of radiologic staging of lung carcinoma can be assessed and compared to mediastinoscopic, bronchoscopic, and surgical staging. If in the opinion of the radiologist insufficient imaging studies have been performed to allow adequate nodal evaluation, box \#320 (Nodes not Assessed") can be checked thus classifying the tumor as NX according to the TNM system.

In the next section on the checklist, the radiologist indicates areas where the reviewed imaging studies suggest metastatic disease and thus the M classification of either M0, M1, or MX under the TNM system can be determined. If the radiologist is of the opinion that the reviewed imaging studies are insufficient to evaluate for
Table 2. Lymph Node Regions to Left of Midline*

1L: Left supraclavicular or scalene nodes (N3).

2L: Left upper paratracheal nodes to the left of the midline of the trachea between the cephalic surface of the aortic arch and the apex of the left lung (N2 if ipsilateral or N3 if contralateral to primary tumor).

4L: Left lower paratracheal nodes to the left of the midline of the trachea between the cephalic surface of the aortic arch and the level of the carina and medial to the ligamentum arteriosum (N2 if ipsilateral, or N3 if contralateral to the primary tumor).

5: Aortopulmonary nodes on left side of mediastinum in the aortopulmonary window, subaortic area, lateral to the aortic arch, lateral to the ligamentum arteriosum and lateral to the main pulmonary artery proximal to the first upper branch of the left pulmonary artery ( $N 2$ if primary tumor in left lung otherwise N3).

6L: Left anterior mediastinal nodes to the left of the midline and anterior to the left subclavian and left common carotid arteries (N2 if ipsilateral, or N3 if contralateral to primary tumor).

8L: Left paraesophageal nodes to left of midline adjacent to the esophagus below region 7 (at least $3 \mathrm{~cm}$ below the carina) and in the left pulmonary ligament (N2 if ipsilateral, or N3 if contralateral to primary tumor).

10L: Peribronchial nodes to the left of the midline of the trachea and along the left main bronchus to the origin of the left upper lobe bronchus (N2 if ipsilateral, or N3 if contralateral to the primary tumor).

11L: Intrapulmonary nodes along the left lobar or segmental bronchi (distal to regions 7 and 10L) which are removed with the left lung specimen (N1 if ipsilateral, or N3 if contralateral to the primary tumor).

14L: Left anterior diaphragmatic nodes adjacent to the diaphragm at the left cardiophrenic angle and middle diaphragmatic area near the phrenic nerve (N2 if ipsilateral, or N3 if contralateral to the primary tumor).

"Modified American Thoracic Society Classification ${ }^{2,10}$

possible metastatic disease, then the tumor can be classified as MX by checking box \#418. Again, a three digit computer code is assigned to each area of metastatic disease. The final section on the checklist allows the radiologist to indicate the types of radiologic examinations that were reviewed and each of these carries a three digit computer code to be entered into the database.

Upon completion of the checklist in a given case, the patient data and the series of three digit codes are entered into the database program using a microcomputer. The program is menu driven to allow use by those with little or no computer background. The command file program consists of a main menu from which the user selects various options including "Add New Patients," "Printer Report," "Edit/Update Existing Database Record," "View Database," and "Exit Lung Carcinoma System." If the "Add 
New Patient" option is requested, the user is asked for the patient ID\# and upon entry of these data the program checks to see if the patient is on the database. The database file is indexed by patient ID\# to allow rapid retrieval of individual patients files. If the patient is already on the database, that file is displayed for editing. If the file is not found, a blank file is displayed for data entry and the new patient is appended to the database. Duplicate entries are thus prevented. If the "Print Report" option is selected, the user is asked for the ID\# and if that patient is on the database, a command file is called that sifts through the three digit codes in that patient file and binds the string variables (from memory) appropriate for that code (eg, 208 ="Right Pleural Effusion $\{T 4\} ")$. A simple logical subroutine determines the radiologic TNM classification and Stage Grouping from the relevant three digit codes. The TNM classification and Stage Grouping are appended to the database each time a report is compiled from a given patient file. Thus, if new data are subsequently added to the file and a new report compiled, the TNM classification and Stage Grouping are automatically updated. The report is printed using the format shown in Fig 2. The core database file contains sufficient fields to store all data from the checklist although additional fields can be added to store user-specific information of inter-

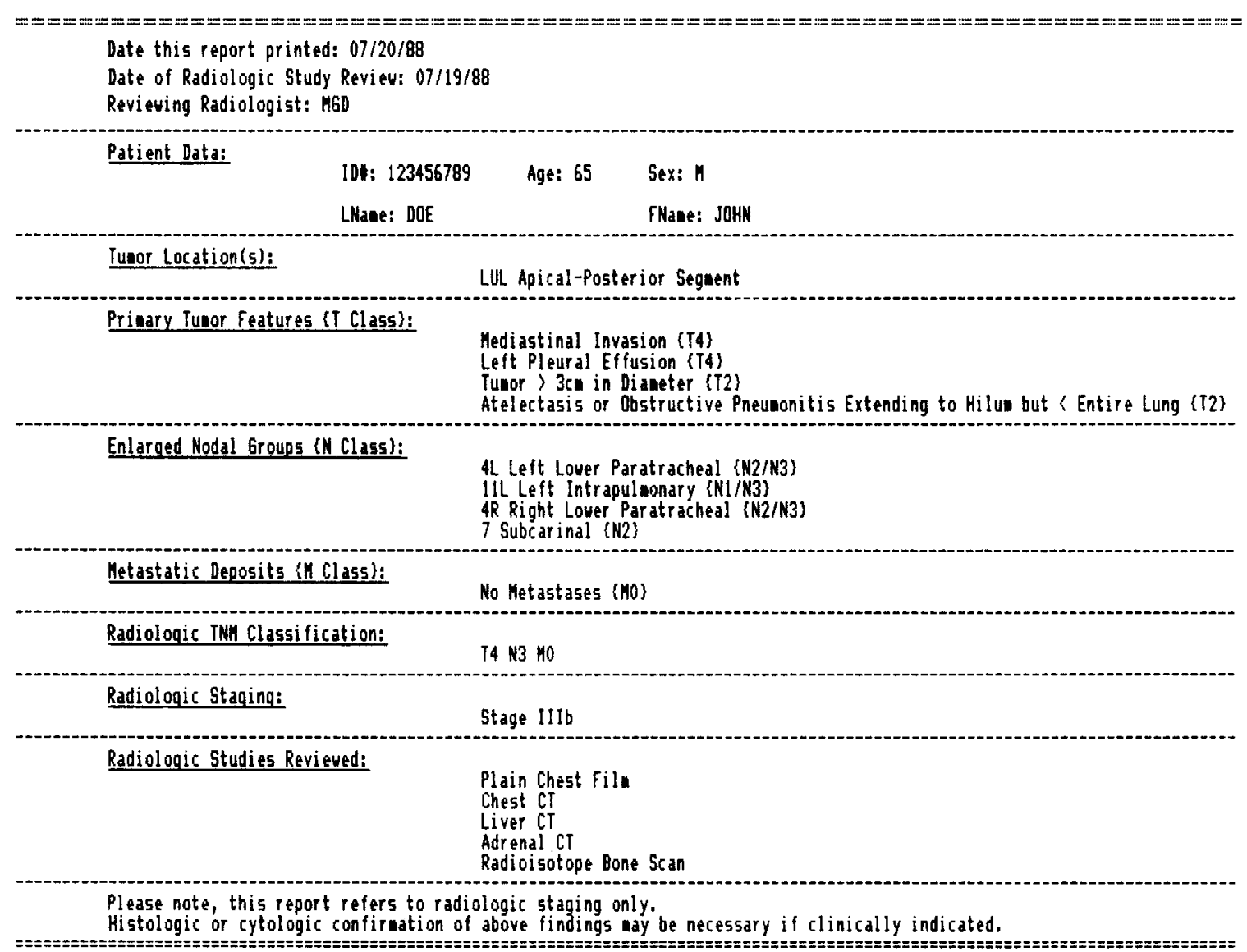

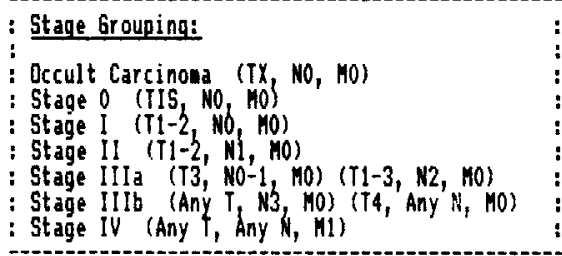

Fig 2. Lung carcinoma report. 
est such as cell type, surgical, radiotherapeutic, or chemotherapeutic data.

As is shown by the disclaimer printed at the bottom of the report (Table 2), the data presented on the report refer to a radiologic staging of the tumor only. Confirmation of the extent of the tumor spread particularly metastases and nodal involvement may be indicated by bronchoscopy, mediastinoscopy, percutaneous biopsy, or surgery. The principal advantage of the checklist/database system is that it encourages the radiologist interpreting the images to apply a systematic and standardized approach and produce more useful informative reports for surgical and medical colleagues. The number and type of radiologic examinations to be routinely performed as part of the workup for carcinoma of the lung will vary depending on the particular institution and referring physician.

Once a significant number of patients have been entered into the database, the user has the opportunity to use the full power of a sophisticated relational database program. The database can be searched for specific groups of patients (eg, all those under 60 years of age with radiologic Stage IIIa who had lower paratracheal adenopathy and pleural effusion). The database can also be used to prepare annual cancer committee reports and monitor how accurately the noninvasive radiologic studies can predict surgical histologic/cytologic staging.

It is hoped that the system decribed will help the radiologist become familiar with the new TNM staging system and provide clinicians with more specific and standardized reports on lung carcinoma patients. Additional checklist/database systems will be developed for application to other tumor types in which radiologic studies play a significant role in clinical staging.

\section{REFERENCES}

1. Mountain CF: Prognostic implications of the international staging system for lung cancer. Semin Oncol 15:236245, 1988

2. Friedman PJ: Lung cancer: Update on staging classifications. AJR 150:261-264, 1988

3. Mountain CF: A new international staging system for lung cancer. Chest 89:225S-233S, 1986

4. Glazer GM, Gross BH, Quint LE, et al: Normal mediastinal lymph nodes: Number and size according to American Thoracic Society mapping. AJR 144:261-265, 1985

5. Glazer GM, Orringer MB, Gross BH, et al: The mediastinum in non-small cell lung cancer: CT-surgical correlation. AJR 142:1101-1105, 1984

6. Friedman PJ, Feigin DS, Liston SE, et al: Sensitivity of chest radiography, computed tomography and gallium scan- ning to metastasis of lung carcinoma. Cancer 54:1300-1306, 1984

7. Baron RL, Levitt RG, Sagel SS, et al: Computed tomography in the preoperative evaluation of bronchogenic carcinoma. Radiology 145:727-732, 1982

8. Schnyder PA, Gamsu G: CT of the pretracheal retrocaval space. AJR 136:303-308, 1981

9. Faling LJ, Pugatch RD, Jung-Legg Y: Computed tomographic scanning of the mediastinum in the staging of bronchogenic carcinoma. Am Rev Respir Dis 124:690-695, 1981

10. Tisi GM, Friedman PJ, Peters RM, et al: Clinical staging of primary lung cancer. Official statement of the American Thoracic Society. Am Rev Respir Dis 127:659. 664,1983 